\title{
Degree-preserving companion of Nelson logic expanded with a consistency operator
}

\author{
F. Esteva $^{1}$, A. Figallo-Orellano ${ }^{2}$, T. Flaminio ${ }^{1}$ and L. Godo ${ }^{1}$ \\ ${ }^{1}$ Artificial Intelligence Research Institute (IIIA) - CSIC, Barcelona, Spain \\ ${ }^{2}$ Departamento de Matemática, Universidad Nacional del Sur (UNS), Bahia Blanca, Argentina and \\ Centre for Logic, Epistemology and The History of Science (CLE) \\ University of Campinas (UNICAMP), Campinas, SP, Brazil
}

\{esteva, tommaso,godo\}@iiia.csic.es, aldofigallo@gmail.com

\begin{abstract}
The main aim of this paper is defining a Logic of Formal Inconsistency over the degree-preserving companion of Nelson logic with a consistency operator. In this sense, we present a quasivariety of Nelson lattices enriched with a suitable consistency operator and axiomatise the corresponding logic. As main results we present necessary and sufficient conditions to prove a categorial equivalence for the category for Nelson lattices with a consistency operator.

Resumo. O principal objetivo desse artigo é definir uma Lógica da Inconcistência Formal sobre a lógica que preserva gaus de verdade que acompanha á lógica de Nelson com um conectivo de consistência. Nesse sentido, apresentamos uma quase-variedade de reticulados de Nelson enriquecidos com um operador de consistência adequado e axiomatizamos a lógica correspondente. Como resultado principal, apresentamos as condições necessárias e suficientes para demonstrar uma equivalência categorial para os reticulados de Nelson com um operador de consistência.
\end{abstract}

\section{Introduction}

In the 1950's, Constructive logic with strong negation (CLSN) was formulated independently by Nelson and Markov as a result of certain philosophical objections to intuitionistic negation. The criticism of intuitionistic negation concerns its disadvantageous nonconstructive property, namely, that the derivability of the formula $\neg(\alpha \wedge \beta)$ in an intuitionistic propositional calculus does not imply that at least one of the formulas $\neg \alpha$ or $\neg \beta$ is derivable.

More recently, in 2008, Spinks and Veroff have shown CLSN, also known in the literature as Nelson logic, can be considered as a substructural logic. More precisely, they have shown that the algebraic models of CLSN, Nelson algebras, are termwise equivalent to certain involutive, bounded, commutative and integral residuated lattices, called Nelson (residuated) lattices, see [6].

The main aim of this paper is defining a Logic of Formal Inconsistency (LFI) based on Nelson logic, by considering the degree-preserving companion of Nelson logic expanded with a consistency operator, in the style of [4]. To do so, we will first algebraically study the quasivariety of Nelson lattices with a consistency operator, then we look at a categorial equivalence between Heyting algebras (with extra structure) and Nelson lattices with consistency operators, and finally we define and axiomatise the logic. 


\section{Preliminaries}

Nelson algebras were introduced by Rasiowa (see, for instance, [7]), under the name of N-lattices, as the algebraic counterpart of the constructive logic with strong negation considered by Nelson and Markov. A Nelson algebra is an algebra $\mathbf{N}=(N, \vee, \wedge, \Rightarrow$ $, \neg, \top, \perp)$ of type $(2,2,2,1,0,0)$ such that $(N, \vee, \wedge, \neg, \top, \perp)$ is a Kleene algebra, and the implication $\Rightarrow$ satisfies the following equations:

$$
\begin{array}{ll}
x \Rightarrow x=\top & x \Rightarrow(y \wedge z)=(x \Rightarrow y) \wedge(x \Rightarrow z) \\
x \wedge(x \Rightarrow y)=x \wedge(\neg x \vee y) & x \Rightarrow(y \Rightarrow z)=(x \wedge y) \Rightarrow z
\end{array}
$$

This definition of Nelson algebras is due to Brignole and Monteiro, and provides an equational characterization of the N-lattices introduced by Rasiowa.

On the other hand, as mentioned above, in [6], the authors have shown that Nelson algebras are termwise equivalent to a certain class of involutive residuated lattices, called Nelson residuated lattices or Nelson lattices. Recall that a commutative, integral, bounded residuated lattice, that we will simply call residuated lattice, is an algebra $\mathcal{A}=\langle A, \wedge, \vee, *, \rightarrow, \perp, \top\rangle$ of type $(2,2,2,2,0,0)$ such that $\langle A, *, \top\rangle$ is a commutative monoid, $\mathcal{L}(A)=\langle A, \wedge, \vee, \perp, \top\rangle$ is a bounded lattice with least element $\top$ and greatest element $\top$, and such that the following condition holds: $x * y \leq z$ iff $x \leq y \rightarrow z$, where $x, y, z$ denote arbitrary elements of $A$ and $\leq$ is the order given by the lattice structure. Since we assume the neutral element of the monoid reduct coincides with the greatest element of $\mathcal{L}(A)$ we have that: $x \leq y$ iff $x \rightarrow y=\top$.

It is well-known that the class $R L$ of residuated lattices is a variety, which is related to different and well-known varieties studied in substructural and fuzzy logics literature. In fact $R L$ coincides with the variety of $\mathrm{FL}_{e w}$-algebras of [5]. We use the denotational conventions of [5]: FL refers to the "Full Lambek calculus", which is the base system and associated algebras, and subindices indicate several axiomatic extensions with properties such as exchange $(e)$ or weakening $(w)$.

A residuated lattice is called involutive if it satisfies the double negation equation: $\neg \neg x=x$, where $\neg x$ is $x \rightarrow \perp$. Besides, it is possible to prove that: $x * y=\neg(x \rightarrow \neg y)$ and $x \rightarrow y=\neg(x * \neg y)$.

A Nelson residuated lattice, or simply Nelson lattice, is an involutive residuated lattice that satisfies the following identity:

$$
(((x * x) \rightarrow y) \wedge((\neg y * \neg y) \rightarrow \neg x)) \rightarrow(x \rightarrow y)=\top .
$$

Nelson lattices will be also called in the paper NL-algebras.

In [2] the authors show that, given a Nelson algebra $\mathbf{N}=(N, \vee, \wedge, \Rightarrow, \neg, \top, \perp)$, then the algebra $\mathcal{R}(\mathbf{N})=(N, \wedge, \vee, *, \rightarrow, \perp, \top)$ is a Nelson residuated lattice, where the operations $*$ and $\rightarrow$ are defined as follows:

$$
x * y:=\neg(x \Rightarrow \neg y) \vee \neg(y \Rightarrow \neg x), \quad x \rightarrow y:=(x \Rightarrow y) \wedge(\neg y \Rightarrow \neg x),
$$

and moreover it holds $\neg x=x \rightarrow \perp$ for each $x \in N$. Conversely, consider now a Nelson lattice $\mathbf{A}=\langle A, \wedge, \vee, *, \rightarrow, \perp, \top\rangle$ and define a binary operation $\rightarrow_{N}$ by specifying $x \rightarrow_{N} y:=(x * x) \rightarrow y$. Then, $\mathcal{N}(A)=\left\langle A, \wedge, \vee, \rightarrow_{N}, \neg, \perp, \top\right\rangle$ is a Nelson algebra.

For a given a Heyting algebra $\mathbf{H}$ and a Boolean filter $F$ of $\mathbf{H}$, let us define $N(H, F):=\{(x, y) \in H \times H: x \wedge y=\perp, x \vee y \in F\}$. Then we have that 
$\mathbf{N}(\mathbf{H}, F)=(N(H, F), \vee, \wedge, \Rightarrow, \neg, * \perp, \top)$ is a Nelson lattice, where the operations are defined as follows:

$$
\begin{gathered}
(x, y) \vee(s, t)=(x \vee s, y \wedge t), \\
(x, y) \wedge(s, t)=(x \wedge s, y \vee t), \\
(x, y) *(s, t)=(x \wedge s,(x \rightarrow t) \wedge(s \rightarrow y)), \\
(x, y) \Rightarrow(s, t)=((x \rightarrow s) \wedge(t \rightarrow y), x \wedge t), \\
\neg(x, y)=(y, x), \\
\top=(\top, \perp), \\
\perp=(\perp, \top) .
\end{gathered}
$$

Finally, we briefly recall the notion a Logic of Formal Inconsistency, LFI for short, see e.g. [3]. Let $\Sigma^{\prime}$ be a propositional signature and assume a denumerable set $\mathcal{V}=$ $\left\{p_{1}, p_{2}, \ldots\right\}$ of propositional variables. The propositional language generated by $\Sigma^{\prime}$ from $\mathcal{V}$ will be denoted by $\mathcal{L}_{\Sigma^{\prime}}$. On the other hand, let $\mathbf{L}=\left\langle\Sigma^{\prime}, \vdash\right\rangle$ be a Tarskian, finitary and structural logic defined over the propositional signature $\Sigma^{\prime}$, which contains a negation $\neg$, and let $\circ$ be a primitive or defined unary connective. Then, $\mathbf{L}$ is said to be a Logic of Formal Inconsistency with respect to $\neg$ and $\circ$ if the following holds:

(i) $\varphi, \neg \varphi \nvdash \psi$, for some $\varphi$ and $\psi$;

(ii) there are two formulas $\alpha$ and $\beta$ such that $\circ \alpha, \alpha \nvdash \beta$ and $\circ \alpha, \neg \alpha \nvdash \beta$;

(iii) $\circ \varphi, \varphi, \neg \varphi \vdash \psi$, for every $\varphi$ and $\psi$.

\section{Nelson residuated lattices with a consistency operator $\circ$}

We start by formally defining the class of Nelson lattices with a consistency operator.

Definition 3.1 $\mathrm{NL}_{\circ}$-algebras are expansions of Nelson lattices with a new unary operation $\circ$ satisfying the following equation and quasi-equation:

(०1) $x \wedge \neg x \wedge \circ(x)=0$

(०2) if $x \wedge \neg x \wedge y=0$ then $y \leq \circ(x)$

In the following, we will use the expression $(\mathbf{A}, \circ)$ to denote the $\mathrm{NL}_{\circ}$-algebra whose Nelson lattice reduct is $\mathbf{A}$.

From this definition, it is clear that the class of $\mathrm{NL}_{\circ}$-algebras is a quasivariety. Now, we have the following easy properties of $\mathrm{NL}_{\circ}$-algebras are displayed in the next lemma.

Lemma 3.2 The following properties hold in a $\mathrm{NL}_{\circ}$-algebra $(\mathbf{A}, \circ)$ :

(i) $\circ x=\max \{z \in A \mid x \wedge \neg x \wedge z=0\}$

(ii) $\circ(x)=\circ(\neg x)=\circ(x \wedge \neg x)=\circ(x \vee \neg x)$

(iii) $\circ(1)=\circ(0)=1$

It follows from (i) of Lemma 3.2 that if a consistency operator is definable in a Nelson lattice it is uniquely determined. Moreover, it also tells us that a consistency operator is always definable in every finite Nelson lattice. However it is not always the case in infinite Nelson lattices as we can see in the next example. 
Example 3.3 Let A be the Nelson lattice over $[0,1] \backslash\left\{\frac{1}{2}\right\}$ with the Nilpotent Minimum operations: $x * y=\min (x, y)$ if $x+y>1$ and $x * y=0$ otherwise; and $\neg x=1-x$. Let $\mathbf{B}$ be the subalgebra of $\mathbf{A} \times \mathbf{A}$ defined on the sublattice consisting of the elements of the form $(x, y)$ with $x, y$ being positive elements of $\mathbf{A}$ (i.e. such that $1 \geq x, y>\frac{1}{2}$ ) and their negations $(1-x, 1-y)$. Take an element $(x, 1) \in B$ such that $1>x>\frac{1}{2}$. An easy computation shows that $\circ((x, 1))$ does not exist.

As is well-known, an implicative filter of a bounded residuated lattice $A$ is a subset $F \subseteq A$ such that $\top \in F$ and it is closed under modus pones: $x \in F$ and $x \rightarrow y \in F$ imply $y \in F$. For each implicative filter $F$, the binary relation $\Theta(F)$ defined by $(x, y) \in \Theta(F)$ if and only if $x \rightarrow y, y \rightarrow x \in F$ is a congruence of the residuated lattice $A$, and $F=\{z \in A:(z, \top) \in \Theta(F)\}$. This is actually a one-one correspondence between the lattice of congruences and the lattice of implicative filters for the variety of bounded residuated lattices. However, since the class of expanded Nelson lattices with a consistency operator involves not only equations but also a quasi-equation, it is a quasivariety. In a quasivariety, congruences that allow for the decomposition of an algebra as a subdirect product of subdirectly irreducible components are required to satisfy an additional condition: the quotient of an algebra by a congruence has to belong to the quasivariety. This condition is automatically satisfied in varieties but not in quasivarieties. Congruences satisfying this condition are usually called Q-congruences. Similarly, filters that are in a one-one correspondence between Q-congruences are implicative filters 'closed' by the quasiequations of the quasivariety, and are called Q-filters.

Therefore, a filter $F$ of a $\mathrm{NL}_{\circ}$-algebra, besides being implicative, has to additionally satisfy the following two conditions:

(F1) if $x \rightarrow y, y \rightarrow x \in F$ then $\circ x \rightarrow \circ y, \circ y \rightarrow \circ x \in F$

(F2) if $x \vee \neg x \vee \neg y \in F$ then $y \rightarrow \circ x \in F$.

We shall call such a filter a o-filter. Note that from (F1) it follows in particular that o-filters are closed by $\circ$ : if $x \in F$ then $\circ x \in F$ as well.

Lemma 3.4 If $(\mathbf{A}, \circ)$ is $\mathrm{NL}_{\circ}$-algebra such that $\mathbf{A}$ is a subdirectly irreducible Nelson lattice, then $\circ$ is such that $\circ(1)=\circ(0)=1$ and $\circ(x)=0$ otherwise, and hence $(\mathbf{A}, \circ)$ is a simple $\mathrm{NL}_{\circ}$-algebra.

In the following, if $\mathbf{A}$ is a Nelson lattice, we will denote by $B(\mathbf{A})$ the set of its Boolean elements, i.e. $B(\mathbf{A})=\{x \in A \mid x \wedge \neg x=0\}$.

Theorem 3.5 Let $(\mathbf{A}, \circ)$ be a subdirectly irreducible $\mathrm{NL}_{\circ}$-algebra. Then $B(\mathbf{A})=\{0,1\}$.

When the algebra is finite, then we can prove more.

Theorem 3.6 Let $(\mathrm{A}, \circ)$ be a finite $\mathrm{NL}_{\circ}$-algebra. Then the following conditions are equivalent:

(i) $(\mathbf{A}, \circ)$ is a s.i. $\mathrm{NL}_{\circ}$-algebra,

(ii) $B(\mathbf{A})=\{0,1\}$,

(iii) $(\mathbf{A}, \circ)$ is a simple $\mathrm{NL}_{\circ}$-algebra.

Theorem 3.7 Let $(\mathrm{A}, \circ)$ be a $\mathrm{NL}_{\circ}$-algebra, then the following conditions are equivalent:

(i) $(\mathbf{A}, \circ)$ is subdirectly irreducible but not simple,

(ii) $B(\mathbf{A})=\{0,1\}$ and there is a non-trivial o-filter $F$ such that $(\circ(x))^{2} \neq 0$ for every $x \in F$. 


\section{A categorial equivalence}

In this section we show an equivalence between a category that involves Heyting algebras (with extra structure) and the algebraic category of Nelson lattices with consistency operators.

For convenience, let us represent $\mathbf{A}$ as $\mathbf{N}(\mathbf{H}, F)$ for $\mathbf{H}$ being a Heyting algebra and $F$ being a Boolean filter of $\mathbf{H}$. So, every $x \in A$ is of the form $(a, b)$ for $a, b \in H$ such that $a \wedge b=\perp$ and $(a \vee b) \in F$. Thus, condition $(i)$ of Lemma 3.2 which indeed characterizes the $\circ$ in a Nelson lattice, can be reformulated in the following way: for all $(a, b) \in A$,

$$
o(a, b)=\max \left\{\left(z, z^{\prime}\right) \in A \mid\left(\perp, a \vee b \vee z^{\prime}\right)=(\perp, \top)\right\}
$$

where the equality holds because $a \wedge b=\perp$ (in $H$ ) for all $(a, b) \in A$. The order on $A$ is defined, with respect to the order of $H$, as $(a, b) \leq(c, d)$ iff $a \leq c$ and $b \geq d$.

Lemma 4.1 For every Heyting algebra $\mathbf{H}$, for every Boolean filter $F$ of $\mathbf{H}$ and for every $a \in H, a \vee \neg a \in F$.

Let us recall from that a unary operation $\ulcorner$ on a Heyting algebra $\mathbf{H}$ is called a dual pseudocomplement, provided that the following equations are satisfied:

(D1) $x \vee\ulcorner(x \vee y)=x \vee\ulcorner y$,

(D2) $x \vee-1=x$,

(D3) $\ulcorner\ulcorner 1=1$.

In every Heyting algebra $\mathbf{H}$ and for every $x \in H$, if is the dual pseudocomplement of $x$ exists, then it is defined as

$$
\ulcorner x=\min \{z \in H \mid x \vee z=1\} .
$$

Proposition 4.2 Let $\mathbf{H}$ be a Heyting algebra, F a Boolean filter of $\mathbf{H}$ and $\mathbf{A}$ the Nelson lattice $\mathbf{N}(\mathbf{H}, F)$. If $\ulcorner(a \vee b)$ exists in $H$, then $\circ(a, b)$ exists and $\circ(a, b)=(\neg d, d)$, where $d=\ulcorner(a \vee b)$. Besides, for any $(a, b) \in A, \circ(a, b) \in A$.

Corollary 4.3 Let $\mathbf{H}$ be a Heyting algebra, $F$ a Boolean filter of $\mathbf{H}$ and $\mathbf{A}$ the Nelson lattice $\mathbf{N}(\mathbf{H}, F)$. Then the operation o exists in $\mathbf{A}$ if and only if the dual pseudo-complement exists for all the elements of $F$. In particular, if $\mathbf{A}=\mathbf{N}(\mathbf{H}, H)$, then $\circ$ exists in $\mathbf{A}$ if and only if the dual pseudo-complementation is definable in the whole $\mathbf{H}$.

\subsection{A categorial equivalence for Nelson lattices with a consistency operator}

To ease the notation, let us denote by $B P F(\mathbf{H})$ the set of the Boolean filters $F$ of a Heyting algebra $\mathbf{H}$ that further satisfy the following property:

(DP) For every $x \in F,\ulcorner x$ exists in $\mathbf{H}$.

Thus, $B P F(\mathbf{H})$ is the set of Boolean filters $F$ of $\mathbf{H}$ such that every element $x \in F$ has a dual pseudocomplement in $\mathbf{H}$.

Definition 4.4 Consider the set $\mathbf{H B P}$ containing pairs $(\mathbf{H}, F)$ such that $\mathbf{H}$ is a Heyting algebra, $F \in B P F(\mathbf{H})$ and maps defined as follows: given two pairs $(\mathbf{H}, F)$ and $\left(\mathbf{H}^{\prime}, F^{\prime}\right)$ a map hetween them is such that:

(m1) h is a Heyting homomorphism between $\mathbf{H}$ and $\mathbf{H}^{\prime}$, 
(m2) $h(F) \subseteq F^{\prime}$,

(m3) for all $x \in F, h\left(\ulcorner x)=\left\ulcorner^{\prime} h(x)\right.\right.$.

It is not difficult to see that HBP is a category and hence we will respectively call objects and morphisms the pairs $(\mathbf{H}, F)$ and the maps $h:(\mathbf{H}, F) \rightarrow\left(\mathbf{H}^{\prime}, F^{\prime}\right)$ of $\mathbf{H B P}$ defined as above.

Moreover, let NC be the algebraic category of Nelson lattices with a consistency operator as in Definition 3.1 and let us consider the map $\mathcal{N C}: \mathbf{H B P} \rightarrow \mathrm{NC}$ defined in the following way:

- For every object $(\mathbf{H}, F) \in \mathbf{H B P}, \mathcal{N C}(\mathbf{H}, F)=(\mathbf{N}(\mathbf{H}, F), \circ)$, where $\mathbf{N}(\mathbf{H}, F)$ is the Nelson algebra of pairs $(a, b) \in H \times H$ such that $a \wedge b=\perp$ and $a \vee b \in F$ as in the above section, and $\circ(a, b)=(\neg\ulcorner(a \vee b),\ulcorner(a \vee b))$ as in Proposition 4.2.

- For every morphism $h:(\mathbf{H}, F) \rightarrow\left(\mathbf{H}^{\prime}, F^{\prime}\right)$ of $\mathbf{H B P}, \mathcal{N C}(h): \mathcal{N C}(\mathbf{H}, F) \rightarrow$ $\mathcal{N C}\left(\mathbf{H}^{\prime}, F^{\prime}\right)$ is so defined: for all $(a, b) \in \mathcal{N C}(\mathbf{H}, F)$,

$$
\mathcal{N C}(h)(a, b)=(h(a), h(b)) .
$$

For every object $(\mathbf{H}, F), \mathcal{N C}(\mathbf{H}, F)$ is an object in NC. Analogously, if $h$ is a morphism of $\mathbf{H B P}, \mathcal{N C}(h)$ is a morphism of NC. The we can prove the following

Proposition 4.5 The map $\mathcal{N C}: \mathrm{HBP} \rightarrow \mathrm{NC}$ is a functor.

Recall that a functor between two categories yields a categorial equivalence iff it is full, faithful and essentially surjective.

Theorem 4.6 The functor $\mathcal{N C}$ establishes a categorial equivalence between $\mathrm{HBP}$ and NC.

\section{Adding a consistency operator to Nelson logic and its paraconsistent companion}

Let $\mathrm{L}$ be the logic of a variety of residuated lattices, that is, an axiomatic extension of $\mathrm{FL}_{e w}$. For each such a logic L, in [1] the authors introduce a companion logic denoted $\mathrm{L} \leq$, whose associated consequence relation has the following semantics: for every set of formulas $\Gamma \cup\{\varphi\}$,

$$
\begin{aligned}
\Gamma \vdash_{\mathrm{L}} \leq \varphi \text { iff } & \text { for every L-algebra } \boldsymbol{A} \text {, every } a \in A, \text { and every } \boldsymbol{A} \text {-evaluation } e, \\
& \text { if } a \leq e(\psi) \text { for every } \psi \in \Gamma \text {, then } a \leq e(\varphi) .
\end{aligned}
$$

For this reason $\mathrm{L}^{\leq}$is known as the degree-preserving companion of $\mathrm{L}$.

Now, let us consider the logic NL corresponding to the variety of Nelson lattices and its degree-preserving companion $\mathrm{NL}^{\leq}$. Since the negation in NL is involutive, and hence it does not satisfy the pseudo-complementation axiom $\varphi \wedge \neg \varphi \rightarrow \overline{0}$, the logic $\mathrm{NL}^{\leq}$ is paraconsitent, i.e. in general

$$
\varphi, \neg \varphi \nvdash_{\mathrm{NL}} \leq \overline{0}
$$

However, $\mathrm{NL}^{\leq}$is not a logic of formal inconstency (LFI) since we cannot define in the language of NL a consistency operator $\circ$ satisfying $\{\circ \varphi, \varphi, \neg \varphi\} \vdash \psi$ for every $\varphi$ and $\psi$. Therefore, we consider next the expansion of NL with a suitable consistency operator $\circ$, that we will call $\mathrm{NL}_{\circ}$, so that its degree-preserving companion $\mathrm{NL}_{\circ}^{\leq}$is a LFI. 
Definition 5.1 We define the logic $\mathrm{NL}_{\circ}$ as the expansion of $\mathrm{NL}$ in a language which incorporates a new unary connective $\circ$ with the following additional axioms and rules:

$$
\begin{array}{ll}
\text { (A1) } & \circ \overline{1} \\
\text { (A2) } \circ \overline{0} \\
\text { (A3) } \neg(\varphi \wedge \neg \varphi \wedge \circ \varphi)
\end{array}
$$

and the following inference rules:

$$
\text { (CNG) } \frac{\varphi \leftrightarrow \psi}{\circ \varphi \leftrightarrow \circ \psi} \quad(\operatorname{Max}) \frac{\varphi \vee \neg \varphi \vee \neg \psi}{\psi \rightarrow \circ \varphi}
$$

Lemma 5.2 The following derivabilities hold in $\mathrm{NL}_{\circ}$ :

i) $\varphi \vee \neg \varphi \vdash_{\mathrm{L}_{\circ}} \circ \varphi$, (ii) $\mathrm{L}_{\circ} \vdash \circ \varphi \rightarrow \neg \varphi \vee \varphi$, (iii) $\varphi \vee \neg \varphi \vdash_{\mathrm{L}_{\circ}} \circ \varphi$.

It is easy to check that, due to the (Cong) rule for $\circ, \mathrm{NL}_{\circ}$ is a Rasiowa implicative logic and hence it is algebraizable. The equivalent algebraic semantics is given by the quasi-variety of $\mathrm{NL}_{\circ}$-algebras. As a direct consequence we have the following general completeness result.

Proposition $5.3 \mathrm{NL}_{\circ}$ is strongly complete w.r.t the class of $\mathrm{NL}_{\circ}$-algebras.

Definition 5.4 The degree-preserving companion of the logic $N L_{\circ}$ is the logic $\mathrm{NL}_{\circ}^{\leq}$defined by the following axioms and rules:

- Axioms of $\mathrm{NL}_{\circ}^{\leq}$are those of $N L_{\circ}$

- Rules of $\mathrm{NL}_{\circ}^{\leq}$are:

(Adj- $\wedge$ ) from $\varphi$ and $\psi$ derive $\varphi \wedge \psi$

(MP- $r$ ) if $\vdash_{\mathrm{NL}_{\circ}} \varphi \rightarrow \psi$, then from $\varphi$ and $\varphi \rightarrow \psi$, derive $\psi$

(CNG- $r$ ) if $\vdash_{\mathrm{NL}_{\circ}} \varphi \leftrightarrow \psi$, then from $\varphi \leftrightarrow \psi$ derive $\circ \varphi \leftrightarrow \circ \psi$

(Max- $r$ ) if $\vdash_{\mathrm{NL}_{\circ}} \neg(\varphi \wedge \neg \varphi \wedge \psi)$, then from $\neg(\varphi \wedge \neg \varphi \wedge \psi)$ derive $\psi \rightarrow \circ \varphi$

It is clear that the degree-preserving companion $\mathrm{NL}_{\circ}^{\leq}$is a LFI.

Let $\mathrm{L}$ be a paraconsistent logic with a consistency operator $\circ$. Then we say that o satisfies the propagation property in $\mathrm{L}$ with respect to a subset $X$ of connectives of the language of $\mathrm{L}$ if $\left\{\circ \varphi_{1}, \ldots, \circ \varphi_{n}\right\} \vdash_{\mathrm{L}} \circ \#\left(\varphi_{1}, \ldots, \varphi_{n}\right)$, for every $n$-ary connective $\# \in X$ and formulas $\varphi_{1}, \ldots, \varphi_{n}$ built with connectives from $X$.

In particular, checking whether $\mathrm{NL}_{\circ}^{\leq}$satisfies the propagation property for the connectives $X=\{\overline{0}, \wedge, \&, \rightarrow\}$ amounts to check the following conditions: $\vdash_{\mathrm{NL}} \circ \overline{0}$ and $\vdash_{\mathrm{NL}}(\circ \varphi \wedge \circ \psi) \rightarrow \circ(\varphi \# \psi)$ for each binary $\# \in X$ (Prop*).

The first condition is obviously satisfied since $\circ \overline{0}$ is an axiom of the logic. Next proposition shows that, in fact, the other conditions are also satisfied.

Proposition 1 NL。 satisfies (Prop*).

In the context of LFIs, it is a desirable property to recover the classical reasoning by means of the consistency connective $\circ$ (see [3]). Specifically, let CPL be classical propositional logic. If $\mathrm{L}$ is a given LFI such that its reduct to the language of CPL is a sublogic of CPL, then a DAT (Derivability Adjustment Theorem) for L with respect to 
CPL is as follows: for every finite set of formulas $\Gamma \cup\{\varphi\}$ in the language of CPL, there exists a finite set of formulas $\Theta$ in the language of $L$, whose variables occur in formulas of $\Gamma \cup\{\varphi\}$, such that:

$$
\text { (DAT) } \Gamma \vdash_{\text {CPL }} \varphi \text { iff } \circ(\Theta) \cup \Gamma \vdash_{\mathrm{L}} \varphi \text {. }
$$

When the operator $\circ$ enjoys the propagation property in the logic L with respect to the classical connectives then the DAT takes the following, simplified form: for every finite set of formulas $\Gamma \cup\{\varphi\}$ in the language of $\mathbf{C P L}$,

$$
\text { (PDAT) } \Gamma \vdash_{\text {CPL }} \varphi \text { iff }\left\{\circ p_{1}, \ldots, \circ p_{m}\right\} \cup \Gamma \vdash_{\mathrm{L}} \varphi
$$

where $\left\{p_{1}, \ldots, p_{m}\right\}$ is the set of propositional variables occurring in $\Gamma \cup\{\varphi\}$.

The fact that $\circ$ has the propagation property it allows us to prove the following PDAT theorem for $\mathrm{NL}_{\circ}^{\leq}$.

Theorem 5.5 (PDAT for $\mathrm{NL}_{\circ}^{\leq}$) Let $\Gamma \cup\{\varphi\}$ be a finite set of formulas in the language of CPL and let $\left\{p_{1}, \ldots, p_{m}\right\}$ the set of propositional variables appearing in $\Gamma \cup\{\varphi\}$. Then

$$
\Gamma \vdash_{\mathbf{C P L}} \varphi \text { iff } \Gamma \vdash_{\mathrm{NL}} \leq\left(\left(\bigwedge_{i=1}^{n} \circ\left(p_{i}\right)\right) *\left(\bigwedge_{i=1}^{n} \circ\left(p_{i}\right)\right)\right) \rightarrow \varphi .
$$

\section{Acknowledgments}

The second author acknowledges support of a post-doctoral grant from FAPESP, Brazil (2016/21928-0). The rest of the authors acknowlege support by the Spanish MINECO/FEDER project RASO (TIN2015- 71799-C2-1-P).

\section{References}

[1] F. Bou, F. Esteva, J. M. Font, A. Gil, L. Godo, A. Torrens and V. Verdú (2009) Logics preserving degrees of truth from varieties of residuated lattices, J. Log. Comput., 19(6):1031-1069

[2] M. Busaniche and R. Cignoli (2010) Constructive Logic with Strong Negation as a Substructural Logic, J. Log. Comput. 20(4): 761-793

[3] W. Carnielli, M. Coniglio, and J. Marcos (2007) Logics of Formal Inconsistency, In Dov Gabbay and Franz Guenthner, editors, Handbook of Philosophical Logic (2nd. edition), volume 14, pages 1-93. Springer.

[4] M.E. Coniglio, F. Esteva and L. Godo (2014) Logics of formal inconsistency arising from systems of fuzzy logic, Logic Journal of the IGPL 22, n. 6: 880-904, 2014.

[5] N. Galatos, P. Jipsen, T. Kowalski, and H. Ono (2007) Residuated Lattices: an Algebraic Glimpseat Substructural Logics, Vol. 151 of Studies in Logic and the Foundations of Mathematics. Elsevier.

[6] M. Spinks and R. Veroff (2008) Constructive logic with strong negation is a substructural logic over FL $\mathrm{F}_{e w}$ I, Studia Logica, 88, 325-348, 2008.

[7] H. Rasiowa (1974) An algebraic approach to non-clasical logics, Studies in logic and the foundations of mathematics, vol. 78. North-Holland Publishing Company, Amsterdam and London, and American Elsevier Publishing Company, Inc., New York. 